\title{
EFICÁCIA DO FLUMIOXAZIN, APLICADO ISOLADO E EM MISTURA COM GLYPHOSATE, PARA O CONTROLE DE PLANTAS DANINHAS EM CITROS
}

\author{
Julio Cezar Durigan ${ }^{(1)}$; Núbia Maria Correia ${ }^{(2)}$; José Antônio Miranda Bellotte ${ }^{(3)}$; Marcos \\ Donizetti Revoredo ${ }^{(3)}$ \\ ${ }^{(1)}$ Prof $^{\mathrm{o}}$ Titular do Departamento de Fitossanidade da FCAV/UNESP. Via de Acesso, Prof. Paulo Donato \\ Castellane, s/n. CEP: 14.884-900. Jaboticabal-SP. E-mail: jcdurigan@fcav.unesp.br ${ }^{(2)} \operatorname{Prof}^{\mathrm{a}} \mathrm{Dr}^{\mathrm{a}}$ do Departamento \\ de Fitossanidade da FCAV/UNESP. ${ }^{(3)}$ Alunos do curso de Pós-Gradução em Produção Vegetal da \\ FCAV/UNESP.
}

RESUMO- Avaliou-se a eficácia do herbicida flumioxazin, aplicado isolado e em mistura com o glyphosate, na cultura de laranja, para controle de Panicum maximum, Cenchrus echinatus, Digitaria horinzotalis, Alternanthera tenella, Bidens pilosa, Malvastrum coromandelianum, Commelina benghalensis, Amaranthus spinosus e Ageratum conyzoides. Os oito tratamentos testados foram: 0,025 e $0,05 \mathrm{~kg} \mathrm{ha}^{-1}$ de flumioxazin (mais óleo mineral a $0,5 \%$ ); $0,025 \mathrm{~kg} \mathrm{ha}^{-1}$ de flumioxazin mais $1,08 \mathrm{~kg}$ e.a. $\mathrm{ha}^{-1}$ de glyphosate (mais óleo mineral a $0,5 \%) ; 0,025 \mathrm{~kg} \mathrm{ha}^{-1}$ de flumioxazin mais $1,44 \mathrm{~kg}$ e.a. ha ${ }^{-1}$ de glyphosate (mais óleo mineral a $0,5 \%) ; 1,08$ e $1,44 \mathrm{~kg}$ e.a. $\mathrm{ha}^{-1}$ de glyphosate, além das testemunhas capinada e infestada. Os herbicidas foram aplicados utilizando-se pulverizador costal manual, à pressão constante $(30 \mathrm{lbf}$ pol- $^{-2}$ ), munido de barra com quatro bicos tipo "leque" XR 110.02 e consumo de calda equivalente a $200 \mathrm{~L} \mathrm{ha}^{-1}$. As avaliações visuais de controle foram realizadas aos 7, 13, 29, 50, 70 e 133 dias após a aplicação, atribuindo-se notas em percentagens. A fitotoxicidade dos herbicidas às plantas cultivadas também foi avaliada. A mistura de flumioxazin com glyphosate, assim como glyphosate isolado, resultou em excelente controle das plantas daninhas, em todas as doses testadas, com exceção de $C$. benghalensis. Não houve benefício da mistura dos herbicidas, nem para $C$. benghalensis que não é bem controlada pelo glyphosate. Os herbicidas não causaram intoxicação às plantas de citros.

Palavras-chave: Flumyzin 500, Roundup, controle químico de plantas daninhas, laranjeira.

\section{EFFICACY OF FLUMIOXAZIN, APPLIED ISOLATED AND MIXTURE WITH GLYPHOSATE, FOR WEED CONTROLIN CITRUS}

\begin{abstract}
It was evaluate the efficacy of the herbicide flumioxazin, applied isolated and in mixture with glyphosate, in orange orchard, for control of Panicum maximum, Cenchrus echinatus, Digitaria horizontalis, Alternanthera tenella, Bidens pilosa, Malvastrum coromandelianum, Commelina benghalensis, Amaranthus spinosus and Ageratum conyzoides. The treatments were: 0.025 and $0.05 \mathrm{~kg} \mathrm{ha}^{-1}$ of flumioxazin (plus mineral oil $0.5 \%$ ); $0.025 \mathrm{~kg}$ $\mathrm{ha}^{-1}$ of flumioxazin plus $1,08 \mathrm{~kg}$ e.a. ha ${ }^{-1}$ of glyphosate (plus mineral oil $0.5 \%$ ); $0.025 \mathrm{~kg} \mathrm{ha}^{-1}$ of flumioxazin plus $1.44 \mathrm{~kg}$ e.a. $\mathrm{ha}^{-1}$ of glyphosate (plus mineral oil $0.5 \%$ ); 1.08 and $1.44 \mathrm{~kg}$ e.a. $\mathrm{ha}^{-1}$ of glyphosate. It was kept two checks: weeded and hand hoed. The herbicides were applied using costal sprayer, constant pressure $\left(30 \mathrm{lbf} \mathrm{pol}^{-2}\right)$, with four nozzles Teejet XR 110.02. The equivalent spray volume was $200 \mathrm{~L} \mathrm{ha}^{-1}$. The weed control evaluations were realized 7, 13, 29, 50, 70 and 133 days after herbicide application, in percentages. It was also evaluated herbicide fitotoxicity toculture. The mixture of flumioxazin with glyphosate, as well as isolated glyphosate, resulted in excellent weed control, in all dosages tested. Not were observed intoxication in citrus plants.
\end{abstract}

Keywords: Flumyzin 500, Roundup, chemical control of weed, orange orchard. 


\section{INTRODUÇÃO}

A citricultura é uma atividade agrícola de grande destaque na economia brasileira, com uma produção estimada em torno de 448 milhões de caixas para a safra 2004/2005, com destaque especial para o Estado de São Paulo, onde se concentra cerca de $72 \%$ das plantas, em área plantada de 587.935 hectares (Agrianual, 2006). Todas as etapas envolvidas na cadeia produtiva dos citros movimentam recursos da ordem de US\$1 bilhão/ano (Neves et al., 2001).

Paralelamente à grande importância econômica representada pela atividade, as plantas de citros são acometidas por uma série de problemas que podem comprometer sua produtividade. Dentre esses entraves, há a interferência das plantas daninhas nos pomares, que podem acarretar perdas de 10 a 50\% (Blanco \& Oliveira, 1978). As plantas daninhas reduzem o crescimento e a produção das plantas cítricas, devido à competição pelos fatores essenciais limitados no ecossistema comum ou, então, pela ação alelopática de substâncias exsudadas pelas mesmas. Além disso, podem servir como hospedeiras intermediárias de pragas e patógenos (Haddad, 1993).

Nas propriedades mais tecnificadas, o controle das plantas daninhas é realizado pela combinação de métodos mecânicos, através da utilização de roçadora nas entrelinhas, e químicos, pela aplicação de herbicidas, em faixas, nas linhas de cultivo (Durigan \& Tiomossi, 2004). Os herbicidas mais utilizados são os aplicados em pós-emergência, destacando-se o glyphosate, paraquat e a mistura de paraquat com diuron. Os produtos de ação residual têm sua utilização limitada principalmente devido ao efeito prejudicial às plantas se não forem aplicados em doses corretas (Haddad, 1993).

Pesquisas relatam que o glyphosate é eficaz no controle de plantas daninhas na cultura dos citros. Sharma (1989) obteve bom controle de Sorghum halepense e Cynodon dactylon e mais de $90 \%$ de controle de Artemisia tridentata, com a utilização desse herbicida. Claus \& Behrens (1976) e Devine et al. (1983) mencionaram que, devido a sua ação sistêmica, o glyphosate ocasionou morte dos rizomas e brotações de Agropyron repens.

Quanto ao flumioxazin, detectou-se eficácia no controle de Amaranthus viridis, Bidens pilosa, Ipomoea grandifolia e Portulaca oleracea, com $25 \mathrm{~g} \mathrm{ha}^{-1}$, e de Acanthosperman hispidum e Digitaria horizontalis, com doses superiores, por até 28 dias após a aplicação, na cultura da cebola (Rozanski et al., 2002). Também nessa cultura, Durigan et al. (2005) obtiveram excelente controle de Lepidium virginicum, até 80 dias após a aplicação, em todas as doses avaliadas (de 80 a $240 \mathrm{~g} \mathrm{ha}^{-1}$ ).

O presente trabalho teve como objetivos avaliar o potencial de controle de plantas daninhas e os possíveis sintomas de intoxicação nas plantas de citros ocasionados pelo flumioxazin, aplicado empós-emergência, isolado e em mistura com o glyphosate.

\section{MATERIAIS E MÉTODOS}

O experimento foi instalado em propriedade particular no município de Bebedouro-SP, de clima Cwa pela classificação Koeppen, apresentando estação seca no inverno. A variedade de laranja utilizada a Valência, enxertada sobre Limão Cravo, com dois anos de idade.

As plantas daninhas presentes na área experimental, as alturas e as suas percentagens de infestação foram: Panicum maximum (50 a $60 \mathrm{~cm}$ de altura, $10 \%$ de infestação), Cenchrus echinatus (40 a $50 \mathrm{~cm}, 16 \%$ de infestação), Digitaria horizontalis (40 a $50 \mathrm{~cm}, 15 \%$ de infestação), Alternanthera tenella (50 cm, 17\% de infestação), Bidens pilosa (20 a $30 \mathrm{~cm}, 8 \%$ de infestação), Malvastrum coromandelianum (40 cm, $8 \%$ de infestação), Commelina benghalensis (30 a 40 cm, 7\% de infestação), Amaranthus spinosus (50 cm, 7\% de infestação) e Ageratum conyzoides (30 cm, 10\% de infestação). 
O delineamento experimental utilizado foi o de blocos ao acaso, com quatro repetições. Os tratamentos testados são apresentados na Tabela 1. As parcelas foram constituídas por duas plantas, com espaçamento de cinco metros entre elas e sete metros entre linhas. As aplicações foram realizadas em dois metros de cada lado da planta por dez metros de comprimento, totalizando $40 \mathrm{~m}^{2}$.

Tabela 1 - Herbicidas e suas doses, além das testemunhas que constituíram os tratamentos avaliados no experimento. Bebedouro-SP, 1998.

\begin{tabular}{|c|c|c|}
\hline \multirow[b]{2}{*}{ Tratamentos } & \multicolumn{2}{|c|}{ Dose do produto } \\
\hline & *i.a. ou e.a. $\left(\mathrm{kg} \mathrm{ha}^{-1}\right)$ & **p.c. $\left(\right.$ kg ou L ha $\left.{ }^{-1}\right)$ \\
\hline Flumioxazin $^{(1)}+$ óleo mineral $^{(2)}$ & $0,025+0,5 \%$ & $0,05+0,5 \%$ \\
\hline Flumioxazin + óleo mineral & $0,05+0,5 \%$ & $0,10+0,5 \%$ \\
\hline Flumioxazin + glyphosate $^{(3)}+$ óleo mineral & $0,025+1,08+0,5 \%$ & $0,05+3,00+0,5 \%$ \\
\hline Flumioxazin + glyphosate + óleo mineral & $0,025+1,44+0,5 \%$ & $0,05+4,00+0,5 \%$ \\
\hline Glyphosate & 1,08 & 3,00 \\
\hline Glyphosate & 1,44 & 4,00 \\
\hline Testemunha capinada & - & - \\
\hline Testemunha sem capina mantida infestada & - & - \\
\hline $\begin{array}{ll}\text { (1) Flumyzin 500 } & * \text { i.a. }=\text { ingrediente ativo } \\
\text { (2) Assist } & * \text { e.a. }=\text { equivalente ácido } \\
\begin{array}{ll}{ }^{(3)} \text { Roundup } & * * \text { p.c. }=\text { produto comercial }\end{array}\end{array}$ & & \\
\hline
\end{tabular}

Os herbicidas foram aplicados em pós-emergência das plantas daninhas, no mês de março de 1998, utilizando-se pulverizador costal manual, à pressão constante (mantida por ar comprimido) de $30 \mathrm{lbf} \mathrm{pol}^{-2}$, munido de barra com quatro bicos de jato plano tipo "leque" XR 110.02, com consumo de calda equivalente a $200 \mathrm{~L} \mathrm{ha}^{-1}$.

No momento da aplicação, a temperatura do ar variou de 32 a $35^{\circ} \mathrm{C}$, a umidade relativa do ar de 70 a $57 \%$, a velocidade dos ventos de 1 a $3 \mathrm{~km} \mathrm{~h}^{-1}$, a nebulosidade de 40 a $50 \%$, a temperatura do solo (a $5 \mathrm{~cm}$ de profundidade) foi de $30^{\circ} \mathrm{C}$ e estava seco na superfície.

As avaliações foram realizadas aos 7, 13, 29, 50, 70 e 133 dias após a aplicação (DAA), exceto para Ageratum conyzoides, que teve o ciclo encerrado antes da última avaliação. Foram realizadas avaliações visuais de controle das plantas daninhas, atribuindo-se notas em percentagens, com base nos procedimentos para instalação, avaliação e análise de experimentos com herbicidas (SOCIEDADE BRASILEIRA DAS CIÊNCIAS DAS PLANTAS DANINHAS, 1995). Além disso, foram observadas possíveis alterações morfológicas e de crescimento nas plantas de citros oriundas da ação dos herbicidas.

Os resultados obtidos foram submetidos ao teste $\mathrm{F}$ da análise de variância. Os efeitos dos herbicidas, quando significativos, foram comparados pelo teste de Tukey à $5 \%$ de probabilidade.

\section{RESULTADOS E DISCUSSÃO}

Ao longo das avaliações, não foram observados sintomas de intoxicação nas plantas de citros, decorrentes dos efeitos dos herbicidas testados, mostrando que os mesmos, nas condições edafo-climáticas vigentes e tecnologia de aplicação utilizada, foram seguros à cultura.

Nas Tabelas de 2 a 10 são apresentados os resultados de controle das plantas daninhas, nas diferentes épocas de avaliações realizadas, cujas interpretações são relatadas a seguir.

O flumioxazin resultou em péssimo controle de P. maximum (Tabela 2). Quando o mesmo foi associado ao glyphosate, obteve-se um desempenho inicial (7 DAA) melhor que o 
glyphosate aplicado isolado. Contudo, a partir dos 14 DAA o controle foi similar entre eles, permanecendo com excelentes notas até a avaliação final. $\mathrm{O}$ controle de A. tenella com flumiozaxin isolado seguiu a mesma tendência observada para $P$. maximum (Tabela 3 ).

Para $C$. echinatus também não houve diferença significativa entre os tratamentos com flumioxazin mais glyphosate e glyphosate isolado, como pode ser observado na Tabela 4. A partir dos 29 DAA foram verificados novos fluxos de emergência de C. echinatus, em todos os tratamentos, indicando ausência de efeito residual do flumioxazin para essa espécie. A aplicação de flumioxazin isolado, independente da dose, resultou em controle não satisfatório.

Até 29 DAA, a percentagem de controle de D. horizontalis (Tabela 5) foi similar para a associação de flumioxazin ao glyphosate e glyphosate isolado. Quando aplicado sozinho, o controle em pós-emergência pelo flumioxazin foi praticamente nulo.

Para $B$. pilosa o controle também foi excelente quando utilizado flumioxazin mais glyphosate ou glyphosate isolado, até 29 DAA (Tabela 6). A partir dessa avaliação ocorreram novos fluxos de emergência em todos os tratamentos testados, resultando em decréscimos expressivos no controle dessa planta daninha.

O controle de $M$. coromandelianum (Tabela 7) foi excelente no início, com a aplicação de flumioxazin, principalmente na sua maior dose. Contudo, diminuiu a partir dos 29 DAA. Houve também melhor desempenho inicial da mistura de flumioxazin com glyphosate comparado ao glyphosate isolado. Notou-se ainda que o flumioxazin apresentou ação residual sobre essa espécie, refletindo substancialmente na percentagem de controle das avaliações finais.

Quanto ao controle de $C$. benghalensis, como pode ser observado na Tabela 8, não foram obtidos bons resultados com flumioxazin mais glyphosate. O controle não foi satisfatório, diminuindo, posteriormente, em virtude da rebrota das plantas. Tanto o glyphosate quanto o flumioxazin, quando aplicados sozinhos, resultaram em baixas percentagens de controle. Não se constataram efeitos sinérgicos dessa mistura para $C$. benghalensis. As plantas apenas tiveram o metabolismo inibido, a parte aérea amarelecida e necrosada, não afetando, no entanto, a sua capacidade de recuperação e reinfestação do local.

Em outro trabalho de pesquisa, a mistura de flumioxazin $\left(60 \mathrm{~g} \mathrm{ha}^{-1}\right)$ com glyphosate $\left(0,72 \mathrm{~kg} \mathrm{ha}^{-1}\right)$ não controlou satisfatoriamente plantas adultas de $C$. benghalensis e $C$. diffusa, refletindo em alto acúmulo de biomassa fresca das plantas ao final de 60 DAA (Ronchi et al., 2002).

A associação de flumioxazin ao glyphosate e glyphosate isolado proporcionaram excelentes resultados de controle de A. spinosus (Tabela 9). O tratamento com flumioxazin a $0,05 \mathrm{~kg} \mathrm{ha}^{-1}$, foi eficaz de 13 a 50 DAA. Todavia, os valores foram estatisticamente inferiores aos da mistura e do glyphosate sozinho. 
Tabela 2 - Percentagens de controle de Panicum maximum na cultura dos citros, em diferentes épocas de avaliação após a aplicação de flumioxazin e glyphosate, isolados e em mistura. Bebedouro-SP, 1998.

\begin{tabular}{|c|c|c|c|c|c|c|c|c|c|c|c|c|c|}
\hline \multirow{2}{*}{ Tratamentos } & \multirow{2}{*}{$\begin{array}{c}\text { Doses }\left(\mathrm{kg} \mathrm{ha}^{-1}\right) \\
\text { i.a. ou e.a. }\end{array}$} & \multicolumn{12}{|c|}{ Época de avaliação do controle (DAA) } \\
\hline & & & 7 & & 13 & & 29 & & $\overline{50}$ & & 70 & 13 & 33 \\
\hline$\overline{\text { Flumioxazin }+ \text { óleo mineral }}$ & $0,025+0,5 \%$ & 5 & $\mathrm{a}^{(1)}$ & 5 & $\mathrm{a}$ & 5 & $\mathrm{a}$ & 5 & $\mathrm{a}$ & & $\mathrm{a}$ & & \\
\hline Flumioxazin + óleo mineral & $0,05+0,5 \%$ & 5 & $\mathrm{a}$ & 5 & $\mathrm{a}$ & 5 & $\mathrm{a}$ & 5 & $\mathrm{a}$ & 5 & $\mathrm{a}$ & $5 \mathrm{a}$ & $\mathrm{a}$ \\
\hline Flumioxazin + glyphosate + óleo mineral & $0,025+1,08+0,5 \%$ & 92,5 & $\mathrm{bc}$ & 100 & $\mathrm{c}$ & 100 & $\mathrm{c}$ & 100 & $\mathrm{c}$ & 100 & $\mathrm{c}$ & $100 \mathrm{c}$ & $\mathrm{c}$ \\
\hline Flumioxazin +glyphosate + óleo mineral & $0,025+1,44+0,5 \%$ & 95,7 & $\mathrm{~cd}$ & 100 & $\mathrm{c}$ & 100 & $\mathrm{c}$ & 100 & $\mathrm{c}$ & 100 & $\mathrm{c}$ & $100 \mathrm{c}$ & $c$ \\
\hline Glyphosate & 1,08 & 88,7 & $\mathrm{~b}$ & 100 & $\mathrm{c}$ & 100 & $\mathrm{c}$ & 100 & $\mathrm{c}$ & 100 & $\mathrm{c}$ & $100 \mathrm{c}$ & $\mathrm{c}$ \\
\hline Glyphosate & 1,44 & 88,7 & $\mathrm{~b}$ & 100 & $\mathrm{c}$ & 100 & $\mathrm{c}$ & 100 & $\mathrm{c}$ & 100 & $\mathrm{c}$ & $100 \mathrm{c}$ & c \\
\hline Testemunha capinada & - & 100 & $\mathrm{~d}$ & 100 & $\mathrm{c}$ & 100 & $\mathrm{c}$ & 100 & $\mathrm{c}$ & 100 & $\mathrm{c}$ & $100 \mathrm{c}$ & c \\
\hline Testemunha sem capina mantida infestada & - & $*(10)$ & $\mathrm{a}$ & $(10)$ & $\mathrm{b}$ & $(10)$ & $\mathrm{b}$ & $(10)$ & $\mathrm{b}$ & (10) & $\mathrm{b}$ & $(10) \mathrm{b}$ & b \\
\hline
\end{tabular}

* Percentagem de cobertura do solo pela comunidade infestante

${ }^{(1)}$ Médias seguidas pela mesma letra não diferem entre si pelo teste de Tukey a 5\% de probabilidade

Tabela 3 - Percentagens de controle de Alternanthera tenella na cultura dos citros, em diferentes épocas de avaliação após a aplicação de flumioxazin e glyphosate, isolados e em mistura. Bebedouro-SP, 1998.

\begin{tabular}{|c|c|c|c|c|c|c|c|c|c|c|c|c|c|}
\hline \multirow{3}{*}{$\begin{array}{r}\text { Tratamentos } \\
\text { Flumioxazin }+ \text { óleo mineral }\end{array}$} & \multirow{2}{*}{$\begin{array}{c}\text { Doses }\left(\mathrm{kg} \mathrm{ha}^{-1}\right) \\
\text { i.a. ou e.a. }\end{array}$} & \multicolumn{12}{|c|}{ Época de avaliação do controle (DAA) } \\
\hline & & \multicolumn{2}{|c|}{07} & \multicolumn{2}{|c|}{13} & \multicolumn{2}{|c|}{29} & \multicolumn{2}{|c|}{50} & \multicolumn{2}{|c|}{70} & \multicolumn{2}{|c|}{133} \\
\hline & $0,025+0,5 \%$ & 11,2 & $\bar{a}$ & 27,5 & $\mathrm{~b}$ & 23,7 & $\mathrm{~b}$ & 15 & $\mathrm{a}$ & 7,5 & $\mathrm{ab}$ & 7,5 & $\mathrm{a}$ \\
\hline Flumioxazin + óleo mineral & $0,05+0,5 \%$ & 15,0 & $\mathrm{ab}$ & 42,5 & $\mathrm{c}$ & 45 & $\mathrm{c}$ & 20 & $\mathrm{a}$ & 6,2 & $\mathrm{a}$ & 7,5 & $\mathrm{a}$ \\
\hline Flumioxazin + glyphosate + óleo mineral & $0,025+1,08+0,5 \%$ & 94,7 & $\mathrm{c}$ & 100 & $\mathrm{~d}$ & 100 & $\mathrm{~d}$ & 99,5 & $\mathrm{~b}$ & 99,5 & $\mathrm{c}$ & 98,7 & $\mathrm{c}$ \\
\hline Flumioxazin + glyphosate + óleo mineral & $0,025+1,44+0,5 \%$ & 97 & $\mathrm{~cd}$ & 100 & $\mathrm{~d}$ & 100 & $\mathrm{~d}$ & 99,5 & $\mathrm{~b}$ & 99,75 & & 93,7 & bc \\
\hline Glyphosate & 1,08 & 93,5 & $\mathrm{c}$ & 100 & $\mathrm{~d}$ & 100 & $\mathrm{~d}$ & 98,5 & $\mathrm{~b}$ & 96 & $\mathrm{c}$ & 83,7 & $\mathrm{~b}$ \\
\hline Glyphosate & 1,44 & 95 & $\mathrm{c}$ & 100 & $\mathrm{~d}$ & 100 & $\mathrm{~d}$ & 98,7 & $\mathrm{~b}$ & 97,5 & $\mathrm{c}$ & 83,7 & $\mathrm{~b}$ \\
\hline Testemunha capinada & - & 100 & $\mathrm{~d}$ & 100 & $\mathrm{~d}$ & 100 & $\mathrm{~d}$ & 100 & $\mathrm{~b}$ & 100 & $\mathrm{c}$ & 100 & $\mathrm{c}$ \\
\hline Testemunha sem capina mantida infestada & - & $*(17,5)$ & $\mathrm{b}$ & $(16,0)$ & $\mathrm{a}$ & $(12,5)$ & $\mathrm{a}$ & $(12,5)$ & $\mathrm{a}$ & $(12,5)$ & $\mathrm{a}$ & $(12,5)$ & $\mathrm{a}$ \\
\hline
\end{tabular}

* Percentagem de cobertura do solo pela comunidade infestante

${ }^{(1)}$ Médias seguidas pela mesma letra não diferem entre si pelo teste de Tukey a 5\% de probabilidade 
Tabela 4 - Percentagens de controle de Cenchrus echinatus na cultura dos citros, em diferentes épocas de avaliação após a aplicação de flumioxazin e glyphosate, isolados e em mistura. Bebedouro-SP, 1998.

\begin{tabular}{|c|c|c|c|c|c|c|c|c|c|c|c|}
\hline \multirow{2}{*}{ Tratamentos } & \multirow{2}{*}{$\begin{array}{c}\text { Doses }\left(\mathrm{kg} \mathrm{ha}^{-1}\right) \\
\text { i.a. ou e.a. }\end{array}$} & \multicolumn{10}{|c|}{ Época de avaliação do controle (DAA) } \\
\hline & & $\mathbf{0 7}$ & & 13 & 29 & & 5 & $\overline{0}$ & 70 & & 133 \\
\hline$\overline{\text { Flumioxazin }+ \text { óleo mineral }}$ & $0,025+0,5 \%$ & $a^{(1)}$ & 13,7 & $\mathrm{a}$ & 20 & $\bar{a}$ & 17,5 & $\mathrm{a}$ & 6,2 & $\bar{a}$ & $7,5 \mathrm{a}$ \\
\hline Flumioxazin + óleo mineral & $0,05+0,5 \%$ & 5 & 17,5 & $\mathrm{a}$ & 17,5 & $\mathrm{a}$ & 15 & $\mathrm{a}$ & 7,5 & $\mathrm{a}$ & 7,5 a \\
\hline Flumioxazin + glyphosate + óleo mineral & $0,025+1,08+0,5 \%$ & $92,5 \mathrm{bc}$ & 100 & $\mathrm{~b}$ & 99 & $\mathrm{~b}$ & 88,7 & $\mathrm{bc}$ & 73,7 & $\mathrm{~b}$ & $52,5 \mathrm{~b}$ \\
\hline Flumioxazin +glyphosate + óleo mineral & $0,025+1,44+0,5 \%$ & $95,75 \mathrm{~cd}$ & 100 & $\mathrm{~b}$ & 98,2 & $\mathrm{~b}$ & 83,7 & $\mathrm{~b}$ & 63,7 & $\mathrm{~b}$ & 55 \\
\hline Glyphosate & 1,08 & $88,75 \mathrm{~b}$ & 100 & $\mathrm{~b}$ & 97,2 & $\mathrm{~b}$ & 85 & $\mathrm{~b}$ & 70 & $\mathrm{~b}$ & $52,5 \mathrm{~b}$ \\
\hline Glyphosate & 1,44 & $88,75 \mathrm{~b}$ & 100 & $\mathrm{~b}$ & 97,5 & $\mathrm{~b}$ & 81,2 & $\mathrm{~b}$ & 60 & $\mathrm{~b}$ & $57,5 \mathrm{~b}$ \\
\hline Testemunha capinada & - & $100 \mathrm{~d}$ & 100 & $\mathrm{~b}$ & 100 & $\mathrm{~b}$ & 100 & $\mathrm{c}$ & 100 & $\mathrm{c}$ & 100 \\
\hline Testemunha sem capina mantida infestada & - & $*(10)$ a & (15) & $\mathrm{a}$ & $(18,7) a$ & $\mathrm{a}$ & $(18,7)$ & $\mathrm{a}$ & $(20,0)$ & $\mathrm{a}$ & $(21,2) \mathrm{a}$ \\
\hline
\end{tabular}

* Percentagem de cobertura do solo pela comunidade infestante

${ }^{(1)}$ Médias seguidas pela mesma letra não diferem entre si pelo teste de Tukey a 5\% de probabilidade

Tabela 5 - Percentagens de controle de Digitaria horizontalis na cultura dos citros, em diferentes épocas de avaliação após a aplicação de flumioxazin e glyphosate, isolados e em mistura. Bebedouro-SP, 1998.

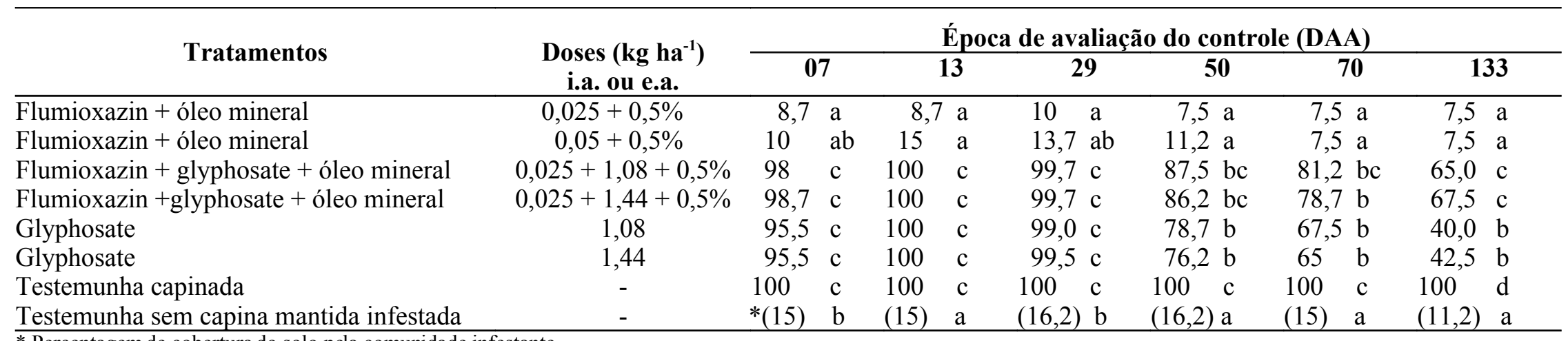

* Percentagem de cobertura do solo pela comunidade infestante

${ }^{(1)}$ Médias seguidas pela mesma letra não diferem entre si pelo teste de Tukey a 5\% de probabilidade 
Tabela 6 - Percentagens de controle de Bidens pilosa na cultura dos citros, em diferentes épocas de avaliação após a aplicação de flumioxazin e glyphosate, isolados e em mistura. Bebedouro-SP, 1998.

\begin{tabular}{|c|c|c|c|c|c|c|c|c|c|c|c|c|c|}
\hline \multirow{2}{*}{ Tratamentos } & \multirow{2}{*}{$\begin{array}{c}\text { Doses }\left(\mathrm{kg} \mathrm{ha}^{-1}\right) \\
\text { i.a. ou e.a. }\end{array}$} & \multicolumn{12}{|c|}{ Época de avaliação do controle (DAA) } \\
\hline & & \multicolumn{2}{|c|}{07} & \multicolumn{2}{|c|}{13} & \multicolumn{2}{|c|}{29} & \multicolumn{2}{|c|}{50} & \multicolumn{2}{|c|}{70} & \multicolumn{2}{|c|}{133} \\
\hline Flumioxazin + óleo mineral & $0,025+0,5 \%$ & 10 & $\mathrm{a}$ & 13,7 & $\mathrm{~b}$ & 11,2 & $a b$ & 11,2 & $\mathrm{a}$ & 6,2 & $\mathrm{a}$ & 7,5 & $\mathrm{a}$ \\
\hline Flumioxazin +óleo mineral & $0,05+0,5 \%$ & 11,2 & $\mathrm{a}$ & 17,5 & $\mathrm{~b}$ & 15 & $\mathrm{~b}$ & 11,2 & $\mathrm{a}$ & 6,2 & $\mathrm{a}$ & 7,5 & $\mathrm{a}$ \\
\hline Flumioxazin +glyphosate + óleo mineral & $0,025+1,08+0,5 \%$ & 100 & $\mathrm{~b}$ & 100 & $\mathrm{c}$ & 99,2 & $\mathrm{c}$ & 65 & $\mathrm{~b}$ & 17,5 & $\mathrm{a}$ & 5,0 & $\mathrm{a}$ \\
\hline Flumioxazin + glyphosate +óleo mineral & $0,025+1,44+0,5 \%$ & 100 & $\mathrm{~b}$ & 100 & $\mathrm{c}$ & 98,5 & $\mathrm{c}$ & 75 & $\mathrm{~b}$ & 17,5 & $\mathrm{a}$ & 7,5 & $\mathrm{a}$ \\
\hline Glyphosate & 1,08 & 100 & $\mathrm{c}$ & 100 & $\mathrm{c}$ & 93,5 & $\mathrm{c}$ & 68,7 & $\mathrm{~b}$ & 22,5 & $\mathrm{a}$ & 6,2 & $\mathrm{a}$ \\
\hline Glyphosate & 1,44 & 100 & $\mathrm{~b}$ & 100 & $\mathrm{c}$ & 98,5 & $\mathrm{c}$ & 65 & $\mathrm{~b}$ & 15,0 & $\mathrm{a}$ & 6,2 & $\mathrm{a}$ \\
\hline Testemunha capinada & - & 100 & $\mathrm{~b}$ & 100 & $\mathrm{c}$ & 100 & $\mathrm{c}$ & 100 & $\mathrm{c}$ & 100 & $\mathrm{~b}$ & 100 & $\mathrm{~b}$ \\
\hline Testemunha sem capina mantida infestada & - & $*(7,5)$ & & $(7,5)$ & $\mathrm{a}$ & $(7,5)$ & $\mathrm{a}$ & $(7,5)$ & $\mathrm{a}$ & $(7,5)$ & $\mathrm{a}$ & $(7,5)$ & $\mathrm{a}$ \\
\hline
\end{tabular}

*Percentagem de cobertura do solo pela comunidade infestante

${ }^{(1)}$ Médias seguidas pela mesma letra não diferem entre si pelo teste de Tukey a $5 \%$ de probabilidade

Tabela 7 - Percentagens de controle de Malvastrum coromandelianum na cultura dos citros, em diferentes épocas de avaliação após a aplicação de flumioxazin e glyphosate, isolados e em mistura. Bebedouro-SP, 1998.

\begin{tabular}{|c|c|c|c|c|c|c|c|c|c|c|}
\hline \multirow{2}{*}{ Tratamentos } & \multirow{2}{*}{$\begin{array}{c}\text { Doses }\left(\mathrm{kg} \mathrm{ha}^{-1}\right) \\
\text { i.a. ou e.a. }\end{array}$} & \multicolumn{9}{|c|}{ Época de avaliação do controle (DAA) } \\
\hline & & & 07 & & 13 & 29 & 5 & 0 & 70 & 133 \\
\hline Flumioxazin + óleo mineral & $0,025+0,5 \%$ & 76,2 & $\mathrm{c}$ & 90 & $\mathrm{a}$ & 70 & 15 & $\mathrm{a}$ & 10 & 25 \\
\hline Flumioxazin + óleo mineral & $0,05+0,5 \%$ & 85 & $\mathrm{~cd}$ & 96,2 & $2 \mathrm{bc}$ & 80 & 50 & $\mathrm{~b}$ & 25 & 30 \\
\hline Flumioxazin + glyphosate + óleo mineral & $0,025+1,08+0,5 \%$ & 90 & de & 100 & $\mathrm{c}$ & $97,5 \mathrm{~d}$ & 90 & de & 75 & 80 \\
\hline Flumioxazin + glyphosate + óleo mineral & $0,025+1,44+0,5 \%$ & 95 & de & 100 & $\mathrm{c}$ & $98,7 \mathrm{~d}$ & 95 & de & 90 & 85 \\
\hline Glyphosate & 1,08 & 50 & $\mathrm{~b}$ & 95 & bc & 90 & 70 & $\mathrm{c}$ & 45 & 50 \\
\hline Glyphosate & 1,44 & 75 & $\mathrm{c}$ & 100 & $\mathrm{c}$ & 100 & 85 & $\mathrm{~d}$ & 50 & 75 \\
\hline Testemunha capinada & - & 100 & $\mathrm{e}$ & 100 & $\mathrm{c}$ & 100 & 100 & $\mathrm{e}$ & 100 & 100 \\
\hline Testemunha sem capina mantida infestada & - & $*(7,5)$ & $\mathrm{a}$ & $(11,2$ & & $(10,0) \mathrm{a}$ & $(10,0)$ & & $(10,0) \mathrm{a}$ & $(10,0) \mathrm{a}$ \\
\hline
\end{tabular}

* Percentagem de cobertura do solo pela comunidade infestante

${ }^{(1)}$ Médias seguidas pela mesma letra não diferem entre si pelo teste de Tukey a 5\% de probabilidade 
Tabela 8 - Percentagens de controle de Commelina benghalensis na cultura dos citros, em diferentes épocas de avaliação após a aplicação de flumioxazin e glyphosate, isolados e em mistura. Bebedouro-SP, 1998.

\begin{tabular}{|c|c|c|c|c|c|c|c|c|c|c|c|c|}
\hline \multirow{2}{*}{ Tratamentos } & \multirow{2}{*}{$\begin{array}{c}\text { Doses }\left(\mathrm{kg} \mathrm{ha}^{-1}\right) \\
\text { i.a. ou e.a. }\end{array}$} & \multicolumn{11}{|c|}{ Época de avaliação do controle (DAA) } \\
\hline & & $\mathbf{0 7}$ & & & 13 & & 29 & 50 & & 70 & & 133 \\
\hline$\overline{\text { Flumioxazin }+ \text { óleo mineral }}$ & $0,025+0,5 \%$ & 12,5 & & 18,7 & $\mathrm{~b}$ & 11,2 & $\mathrm{a}$ & $16,25 \mathrm{ab}$ & 5 & $\mathrm{a}$ & 5 & $\mathrm{a}$ \\
\hline Flumioxazin + óleo mineral & $0,05+0,5 \%$ & 13,7 & $\mathrm{a}$ & 16,2 & $a b$ & 11,2 & $\mathrm{a}$ & 5 & 5 & $\mathrm{a}$ & 5 & $\mathrm{a}$ \\
\hline Flumioxazin + glyphosate + óleo mineral & $0,025+1,08+0,5 \%$ & 40 & $\mathrm{~b}$ & 60 & $\mathrm{c}$ & 82,5 & $\mathrm{c}$ & $\mathrm{bc}$ & 41,2 & $a b$ & 13,7 & $\mathrm{a}$ \\
\hline Flumioxazin +glyphosate + óleo mineral & $0,025+1,44+0,5 \%$ & 42,5 & $\mathrm{~b}$ & 62,5 & $\mathrm{c}$ & 86,2 & $\mathrm{c}$ & $67,5 \mathrm{~cd}$ & 60 & $\mathrm{bc}$ & 12,5 & $\mathrm{a}$ \\
\hline Glyphosate & 1,08 & 12,5 & a & 12,5 & $a b$ & 38,7 & $\mathrm{~b}$ & 43,7 bc & 22,5 & $a b$ & 15 & $\mathrm{a}$ \\
\hline Glyphosate & 1,44 & 11,2 & $\mathrm{a}$ & 12,5 & $a b$ & 38,7 & $\mathrm{~b}$ & $37,5 \mathrm{abc}$ & 16,2 & $a b$ & 15 & $\mathrm{a}$ \\
\hline Testemunha capinada & - & 100 & $\mathrm{c}$ & 100 & $\mathrm{~d}$ & 100 & $\mathrm{c}$ & 100 & 100 & $\mathrm{c}$ & 100 & $\mathrm{~b}$ \\
\hline Testemunha sem capina mantida infestada & - & $*(6,2)$ & & $(7,5)$ & $\mathrm{a}$ & $(7,5)$ & $\mathrm{a}$ & $(7,5) \quad a$ & $(7,5)$ & $\mathrm{a}$ & $(6,2)$ & $\mathrm{a}$ \\
\hline
\end{tabular}

* Percentagem de cobertura do solo pela comunidade infestante

${ }^{(1)}$ Médias seguidas pela mesma lera não diferem entre si pelo teste de Tukey a 5\% de probabilidade

Tabela 9 - Percentagens de controle de Amaranthus spinosus na cultura dos citros, em diferentes épocas de avaliação após a aplicação de flumioxazin e glyphosate, isolados e em mistura. Bebedouro-SP, 1998.

\begin{tabular}{|c|c|c|c|c|c|c|c|c|c|c|c|c|}
\hline \multirow{2}{*}{ Tratamentos } & \multirow{2}{*}{$\begin{array}{c}\text { Doses }\left(\mathrm{kg} \mathrm{ha}^{-1}\right) \\
\text { i.a. ou e.a. }\end{array}$} & \multicolumn{11}{|c|}{ Época de avaliação do controle (DAA) } \\
\hline & & & 07 & & 13 & & 29 & & 50 & 7 & $\mathbf{0}$ & 133 \\
\hline Flumioxazin + óleo mineral & $0,025+0,5 \%$ & 20 & $\mathrm{~b}$ & 25 & $\mathrm{~b}$ & 70 & $\mathrm{~b}$ & 50 & $\mathrm{~b}$ & 50 & $\mathrm{~b}$ & 45 \\
\hline Flumioxazin + óleo mineral & $0,05+0,5 \%$ & 60 & $\mathrm{c}$ & 70 & $\mathrm{c}$ & 90 & $\mathrm{c}$ & 85 & $\mathrm{c}$ & 65 & $\mathrm{c}$ & 65 \\
\hline Flumioxazin + glyphosate + óleo mineral & $0,025+1,08+0,5 \%$ & 98 & $\mathrm{~d}$ & 100 & $\mathrm{~d}$ & 100 & $\mathrm{~d}$ & 100 & $\mathrm{~d}$ & 95 & $\mathrm{~d}$ & 95 \\
\hline Flumioxazin +glyphosate + óleo mineral & $0,025+1,44+0,5 \%$ & 99 & $\mathrm{~d}$ & 100 & $\mathrm{~d}$ & 100 & $\mathrm{~d}$ & 100 & $\mathrm{~d}$ & 98,7 & $\mathrm{~d}$ & $97,5 \mathrm{~d}$ \\
\hline Glyphosate & 1,08 & 90 & $\mathrm{~d}$ & 100 & $\mathrm{~d}$ & 100 & $\mathrm{~d}$ & 95 & $\mathrm{~d}$ & 98,7 & $\mathrm{~d}$ & $95 \mathrm{~d}$ \\
\hline Glyphosate & 1,44 & 95 & $\mathrm{~d}$ & 100 & $\mathrm{~d}$ & 100 & $\mathrm{~d}$ & 95 & $\mathrm{~d}$ & 98,7 & $\mathrm{~d}$ & 95 \\
\hline Testemunha capinada & - & 100 & $\mathrm{~d}$ & 100 & $\mathrm{~d}$ & 100 & $\mathrm{~d}$ & 100 & $\mathrm{~d}$ & 100 & $\mathrm{~d}$ & 100 \\
\hline Testemunha sem capina mantida infestada & - & $*(6,2)$ & $\mathrm{a}$ & $(7,5)$ & $\mathrm{a}$ & $(7,5)$ & $\mathrm{a}$ & $(7,5)$ & $\mathrm{a}$ & $(7,5)$ & $\mathrm{a}$ & $(7,5) \quad a$ \\
\hline
\end{tabular}

* Percentagem de cobertura do solo pela comunidade infestante

${ }^{(1)}$ Médias seguidas pela mesma letra não diferem entre si pelo teste de Tukey a $5 \%$ de probabilidade 
Tabela 10 - Percentagens de controle de Ageratum conyzoides na cultura dos citros, em diferentes épocas de avaliação após a aplicação de flumioxazin e glyphosate, isolados e em mistura. Bebedouro-SP, 1998.

\begin{tabular}{|c|c|c|c|c|c|c|}
\hline \multirow{2}{*}{ Tratamentos } & \multirow{2}{*}{$\begin{array}{c}\text { Doses }\left(\mathrm{kg} \mathrm{ha}^{-1}\right) \\
\text { i.a. ou e.a. }\end{array}$} & \multicolumn{5}{|c|}{ Época de avaliação do controle (DAA) } \\
\hline & & 07 & 13 & 29 & 50 & 70 \\
\hline Flumioxazin + óleo mineral & $0,025+0,5 \%$ & $25 \mathrm{ab}$ & $30 \mathrm{~b}$ & $70 \mathrm{~b}$ & $60 \mathrm{~b}$ & $60 \mathrm{~b}$ \\
\hline Flumioxazin + óleo mineral & $0,05+0,5 \%$ & $30 \mathrm{~b}$ & $40 \mathrm{c}$ & $75 \mathrm{~b}$ & $85 \mathrm{c}$ & $75 \mathrm{c}$ \\
\hline Flumioxazin + glyphosate + óleo mineral & $0,025+1,08+0,5 \%$ & $75 \mathrm{c}$ & $100 \mathrm{~d}$ & $100 \mathrm{c}$ & $100 \mathrm{~d}$ & $100 \mathrm{~d}$ \\
\hline Flumioxazin + glyphosate + óleo mineral & $0,025+1,44+0,5 \%$ & $92,5 \mathrm{e}$ & $100 \mathrm{~d}$ & $100 \mathrm{c}$ & $100 \mathrm{~d}$ & $100 \mathrm{~d}$ \\
\hline Glyphosate & 1,08 & $50 \mathrm{c}$ & $100 \mathrm{~d}$ & $100 \mathrm{c}$ & $100 \mathrm{~d}$ & $100 \mathrm{~d}$ \\
\hline Glyphosate & 1,44 & $60 \mathrm{~cd}$ & $100 \mathrm{~d}$ & $100 \mathrm{c}$ & $100 \mathrm{~d}$ & $100 \mathrm{~d}$ \\
\hline Testemunha capinada & - & $100 \mathrm{e}$ & $100 \mathrm{~d}$ & $100 \mathrm{c}$ & $100 \mathrm{~d}$ & $100 \mathrm{~d}$ \\
\hline Testemunha sem capina mantida infestada & - & $*(10) \quad \mathrm{a}$ & (10) a & (10) a & $(8,7) \mathrm{a}$ & $(8,7) \mathrm{a}$ \\
\hline
\end{tabular}

* Percentagem de cobertura do solo pela comunidade infestante

$(8,7) \mathrm{a}$

${ }^{(1)}$ Médias seguidas pela mesma lđra não diferem entre si pelo teste de Tukey a 5\% de probabilidade. 
O controle de $A$. conyzoides (Tabela 10) foi similar ao observado para A. spinosus. Constatou-se alta percentagem de controle dessa espécie com a aplicação de flumioxazin mais glyphosate e glyphosate isolado. A aplicação isolada de flumioxazin, na maior dose, proporcionou bons resultados nas avaliações de 29 a 70 DAA. Essa espécie não foi avaliada aos 133 DAA, pois as plantas encerraram o seu ciclo de vida, tornando-se ausentes na área experimental, inclusive na testemunha sem capina mantida infestada.

Para as condições edafo-climáticas em que se desenvolveu o experimento, percebeu-se que a associação de flumioxazin ao glyphosate, assim como glyphosate sozinho, em todas as doses testadas, foram eficazes no controle de $P$. maximum, $C$. echinatus, $D$. horizontalis, $A$. tenella, B. pilosa, M. coromandelianum, A. spinosus e A. conyzoides. No entanto, não houve benefício da mistura dos herbicidas em relação à aplicação de glyphosate sozinho.

Vários autores relatam a efetividade da utilização de glyphosate sozinho ou associado a outro herbicida no controle de diferentes plantas daninhas. Assim, Monquero et al. (2001), testaram, dentre outras misturas, glyphosate associado ao flumioxazin para o controle de Richardia brasiliensis, Commelina benghalensis, Amaranthus hybridus, Galinsoga parviflora e Ipomoea grandifolia, em experimento conduzido em condições de casa de vegetação, obtendo excelentes resultados. Da mesma forma, a mistura em tanque de glyphosate com flumioxazin resultou em bom controle de Ipomoea spp. na cultura do algodoeiro (Wright et al., 2000). Sobre a ação da combinação de herbicidas na cultura dos citros, Josan et al. (1994), utilizando glyphosate sozinho ou associado ao diuron, obtiveram redução de aproximadamente $80,2 \%$ de monocotiledôneas e $82,3 \%$ de dicotiledôneas, sem nenhum efeito fitotóxico às plantas cultivadas.

O flumioxazin sozinho, independente da dose testada, resultou em péssimo controle das plantas daninhas. Com exceção de $M$. coromandelianum que foi controlada razoavelmente pelo herbicida até os $29 \mathrm{DAA}$. Os resultados obtidos podem ser atribuídos ao estádio de desenvolvimento das plantas no momento da aplicação, às doses utilizadas e a própria fisiologia do herbicida. O flumioxazin é absorvido rapidamente pelas plantas, porém, devido ao seu mecanismo de ação, possui translocação limitada, pois interage instantaneamente com as células do parênquima restringindo a sua movimentação na planta (Merotto Junior \& Vidal, 2001). Conseqüentemente, o herbicida não atinge o sistema radicular ou mesmo o dossel completo das plantas, favorecendo a recuperação e a rebrota das mesmas.

Dessa forma, pode-se concluir que houve excelente controle das plantas daninhas, em pós-emergência, para a mistura de flumioxazin mais glyphosate e glyphosate isolado, em todas as doses testadas. A mistura dos herbicidas não beneficiou o controle das plantas daninhas, inclusive de Commelina benghalensis. A aplicação de flumioxazin, na dose de $0,05 \mathrm{~kg} \mathrm{ha}^{-1}$, destacou-se para o controle em pós-emergência de Malvastrum coromandelianum, até 29 DAA, e de Amaranthus spinosus e Ageratum conyzoides, até 70 DAA. O flumioxazin mostrou alguma ação residual para Digitaria horizontalis, Alternanthera tenella e Malvastrum coromandelianum. Os herbicidas não ocasionaram sintomas de intoxicação às plantas de citros.

\section{LITERATURA CITADA}

AGRIANUAL 2006: Anuário da agricultura brasileira. São Paulo: Instituto FNP, 2006. $504 \mathrm{p}$.

BLANCO, H.G.; OLIVEIRA, D.A. Estudos dos efeitos da época de controle do mato sobre a produção de citros e a composição da flora daninha. Arquivo Instituto Biológico de São Paulo, São Paulo-SP, v.45, n.1, p.25-26, 1978.

CLAUS, J.S.; BEHRENS, R. Glyphosate translocation and quackgrass rhizome bud kill. Weed Science, Champaign, v.24, n.2, p.149-152,1976. 
DEVINE, M.D.; BANDEEN, J.D.; MCKERSIE, B.D. Temperature effects on glyphosate absorption, translocation and distribution in quackgrass (Agropyron repens). Weed Science, Champaign, v.31, n.4,p.461-464, 1983.

DURIGAN, J.C.; TIMOSSI, P.C. Manejo de plantas daninhas em pomares cítricos. Bebedouro: EECB,n.22, 2002. 53p. ( Boletim Citrícola)

DURIGAN, J.C.; SILVA. M.R.M.; AZANIA, A.A.P.M. Eficácia e seletividade do herbicida flumioxazin aplicado em pré-emergência na cultura transplantada da cebola. Revista Brasileira de Herbicidas, v.4, n.3, p.11-17, 2005.

HADDAD, A.C. Racionalização de herbicidas aplicados ao solo e em pós-emergência das plantas daninhas em pomares cítricos (Citrus sinensis (L.) osbeck). 1993. f. 4-23. Monografia (Trabalho de Graduação em Agronomia) - Faculdade de Ciências Agrárias e Veterinárias, Universidade Estadual Paulista, Jaboticabal, 1993.

JOSAN, J.S.; THATAI, S.K.; MONGA, P.K.; SHARMA, J.N. Effect of various herbicides on weed control in citrus. Journal of Research, Punjab, v.31, n.4, p.402-406, 1994.

MEROTTO JUNIOR, A.; VIDAL, R.A. Herbicidas inibidores de PROTOX. In: VIDAL, R.A.; MEROTTO JUNIOR, A. (Ed.) Herbicidologia. 1를. ed., 2001. p.69-86

MONQUERO, P.A.; CHRISTOFFOLETI, P.J.; SANTOS, C.T.D. Glyphosate combined with alternative herbicides for vegetation management. Planta Daninha, Brasília-DF, v.19, n.3, p.375-380, 2001.

NEVES, E.M.; DAYOB, M.; DRAGONE, D.S.; NEVES, M.F. Citricultura brasileira - efeitos econômicos-financeiros, 1996-2000. Revista Brasileira de Fruticultura, Jaboticabal-SP, v.23, n.2, p.432-436, 2001.

RONCHI, C.P.; SILVA, A.A.; MIRANDA, G.V.; FERREIRA, L.R.; TERRA, A.A. Eficência de misturas para o controle de plantas daninhas do gênero Commelina. Planta Daninha, Viçosa-MG, v.20, n.2, p.311-318, 2002.

ROZANSKI, A.; COSTA, E.A.D. da; MATALLO, M.B.; BURGA, C.A. Efeito do herbicida flumioxazin nas plantas daninhas e na cultura da cebola. Disponível em $<\underline{\text { http://www.abhorticultura.com.br/biblioteca/Default.asp? } \mathbf{d}=3284>}$ Acesso em: 28 de setembro de 2006.

SHARMA, K.K. Control of weeds in citrus orchards with Roundup, Gramoxone and 2,4-D. Journal of Research, Punjab, v.26, n.3, p.407-409, 1989.

SOCIEDADE BRASILEIRA DAS CIÊNCIAS DAS PLANTAS DANINHAS. Procedimentos para instalação, avaliação e análise de experimentos com herbicidas. Londrina: SBCPD, 1995. 42p.

WRIGHT, S.D.; VARGAS, R.N.; BANUELOS, G.; MARTIN-DUVAL, T.; DUGGER, P.; RICHTER, D. Annual morningglory (Ipomoea spp.) control in cotton. Proceedings Beltwide Cotton Conferences, San Antonio, v.2, p.1465-1467, 2000. 